\title{
Reciprocal Modulatory Influences between Tonotopic and Nontonotopic Cortical Fields in the Cat
}

\author{
Andres Carrasco ${ }^{1}$ and Stephen G. Lomber ${ }^{2,3}$ \\ ${ }^{1}$ Graduate Program in Neuroscience, University of Western Ontario, London, Ontario N6A 5B8, Canada, ${ }^{2}$ Centre for Brain and Mind, Department of \\ Physiology and Pharmacology, Schulich School of Medicine and Dentistry, University of Western Ontario, London, Ontario N6A 5C1, Canada, and \\ ${ }^{3}$ Cerebral Systems Laboratory, Department of Psychology, Faculty of Social Science, University of Western Ontario, London, Ontario N6A 5C2, Canada
}

Functional and anatomical studies suggest that acoustic signals are processed hierarchically in auditory cortex. Although most regions of acoustically responsive cortex are not tonotopically organized, all previous electrophysiological investigations of interfield interactions have only examined tonotopically represented areas. The purpose of the present study was to investigate the functional interactions between tonotopically and nontonotopically organized fields in auditory cortex. We accomplished this goal by examining the bidirectional contributions between the cochleotopically organized primary auditory cortex (A1) and the noncochleotopically organized second auditory field (A2). Multiunit acute recording procedures in combination with reversible cooling deactivation techniques were used in eight mature cats. The synaptic activity of A1 or A2 was suppressed while the neuronal response to tonal stimuli of the noninactivated area (A1 or A2) was measured. Response strength, neuronal threshold, receptive field bandwidths, and latency measures were collected at each recorded site before, during, and after cooling deactivation epochs. Our analysis revealed comparable changes in A1 and A2 neuronal response properties. Specifically, significant decreases in neuronal response strength, increases in neuronal threshold, and shortening of response latency were found in both fields during periods of cooling deactivation. The weak anatomical connections between the two fields investigated make these findings unexpected. Furthermore, the observed neuronal changes suggest a model of corticocortical interaction among auditory fields in which neither differences in the magnitude of anatomical projections nor cortical representation of sensory stimuli are reliable determinants of modulatory functions.

\section{Introduction}

Models of hierarchical processing in the cerebrum have been substantiated by numerous studies in the visual, auditory, and somatosensory systems of primates, felines, and rodents (Van Essen et al., 1990; Rouiller et al., 1991; Pons et al., 1992; Coogan and Burkhalter, 1993; Wessinger et al., 2001). Functionally, three lines of evidence have been provided to support the proposed schemes. First, disruption of neuronal activity in early processing regions of cortex has a direct effect on the response properties of "higher" order modules (Girard and Bullier, 1989; Girard et al., 1991a,b; Pons et al., 1992; Rauschecker et al., 1997; Carrasco and Lomber, 2009b). Second, increased neuronal response latency to sensory stimulation is conspicuous with ascension in the hierarchical pathway (Schmolesky et al., 1998; Bullier, 2001). Third, neuronal selectivity to sensory features increases with progression among regions of the suggested model (Rodman and Albright, 1989; Wessinger et al., 2001). In addition to functional studies, neuroanatomical investigations have provided evidence for the existence of hierarchical sensory processing in cortex

\footnotetext{
Received Nov. 17, 2009; accepted Dec. 5, 2009.

This work was supported in part by grants from the Canadian Institutes of Health Research, the Natural Sciences and Engineering Research Council of Canada, and the Canada Foundation for Innovation.

Correspondence should be addressed to Stephen G. Lomber, Cerebral Systems Laboratory, Department of Physiology and Pharmacology, M216 Medical Sciences Building, University of Western Ontario, London, 0N N6A 5C1, Canada. E-mail: steve.lomber@uwo.ca.

DOI:10.1523/JNEUROSC1.5708-09.2009

Copyright $\odot 2010$ the authors $\quad 0270-6474 / 10 / 301476-12 \$ 15.00 / 0$
}

(Amir et al., 1993; Kaas and Hackett, 1999). Consequently, functional and anatomical studies have validated the presence of hierarchically organized processing modules in the cerebrum.

Wide acceptance of hierarchical organization in sensory cortex has fomented investigations into the existence of "streams of information," in which features of sensory signals are processed by separate cortical modules acting in concert. Relevant to the present investigation is evidence for the emergence of "what" and "where" streams of information processing in auditory cortex (Rauschecker et al., 1997; Kaas and Hackett, 1999; Lomber and Malhotra, 2008). Although these investigations have provided support for specialized processing pathways, to date, every electrophysiological investigation of this model has been limited to the study of interactions between tonotopically organized fields (Kitzes and Hollrigel, 1996; Rauschecker et al., 1997; Carrasco and Lomber, 2009a,b). As the majority of fields that form auditory cortex are nontonotopically organized (Reale and Imig, 1980; Kosaki et al., 1997), omission of these areas in previous investigations has resulted in a limited insight into interfield functional influences. In an effort to further our understanding of communicative properties between cortical areas of auditory cortex, the present study examined the functional properties between tonotopically and nontonotopically represented cortical fields.

We investigated the bidirectional functional interactions between the tonotopically organized primary auditory cortex (A1) and the nontonotopically organized second auditory field (A2) of 


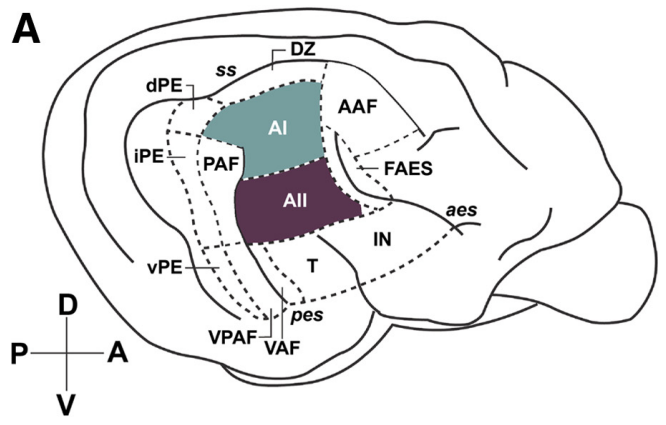

B

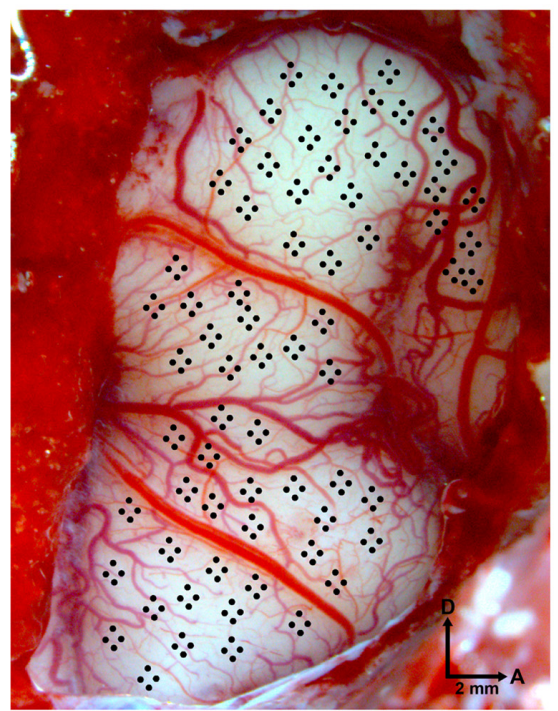

C

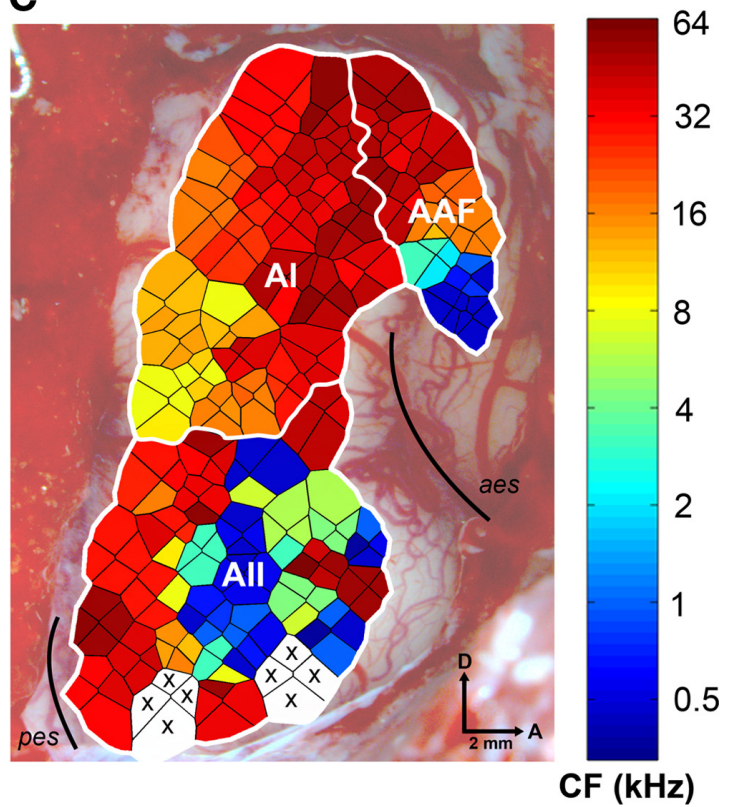

Figure 1. Lateral view of the right hemisphere of the cat cerebral cortex showing the loci examined. $A$, Schematic drawing of the 13 areas of cat auditory cortex. The highlighted regions illustrate the two areas examined in this study. $\boldsymbol{B}$, Photomicrograph of the right hemisphere of a cat showing the location of individual microelectrode penetrations and their relationship to cortical vasculature. Each black dot in the figure represents a single recording site. C, Characteristic frequency organization of three auditory cortical fields. The borders (white lines) were defined based on tonotopic organization and latency characteristics. Each polygon represents an estimation of the cortical area with similar response properties as the recorded site. The polygon colors correspond to the characteristic frequency identified at each site. The the cat. Based on neuroanatomical studies, we hypothesized that the weak projections from A1 to A2 (Lee and Winer, 2008) would result in small changes in $\mathrm{A} 2$ neuronal response properties during A1 deactivation. Conversely, the larger number of projections from A2 to A1 would substantially influence the response properties of A1 neurons during A2 deactivation (Lee and Winer, 2008). However, the results did not support our hypothesis and demonstrated a comparable reduction in response strength in both fields during epochs of deactivation. These findings provide evidence that, although feedforward and feedback connections between tonotopically and nontonotopically organized cortical fields can differ in arrangement and magnitude, they can have comparable modulatory influences.

\section{Materials and Methods}

Overview. Neuronal activity in the right auditory cortex (Fig. 1A) of eight adult cats was measured during periods of acoustic stimulation. All procedures conducted were approved by the University of Western Ontario Animal Use Subcommittee of the University Council on Animal Care and were implemented in accordance to the National Research Council's Guidelines for the Care and Use of Mammals in Neuroscience and Behavioral Research (2003) and the Canadian Council on Animal Care's Guide to the Care and Use of Experimental Animals (Olfert et al., 1993). The detailed methodology used in this investigation has been described by Carrasco and Lomber (2009a).

Surgical preparation. Cats were anesthetized with sodium pentobarbital (25 mg/kg, i.v.) (Cheung et al., 2001), and levels of anesthesia were monitored using electrocardiogram and blood oxygen concentration levels. Supplemental doses of sodium pentobarbital were administered as needed. Body temperature was monitored with a rectal probe. The incidence of edema and respiratory secretions was reduced by administering dexamethasone and atropine $(0.03 \mathrm{mg} / \mathrm{kg}$, s.c.) on a $12 \mathrm{~h}$ schedule. An infusion pump hydrated the animal by supplying $2.5 \%$ dextrose/halfstrength lactated Ringer's solution $\left(4 \mathrm{ml} \cdot \mathrm{kg}^{-1} \cdot \mathrm{h}^{-1}\right.$, i.v. $)$. Animals were intubated with a cuffed endotracheal tube. Respiration was unassisted. A craniotomy was performed over areas $\mathrm{A} 1$ and $\mathrm{A} 2$ of the right hemisphere, and the dura was resected. Desiccation was prevented by applying a layer of silicone oil to the pial surface. A picture of the exposed cortical region was taken to maintain a record of the location of each electrode penetration (Fig. $1 B$ ).

Stimulus generation and presentation. Receptive fields were constructed by presenting 2064 pure tones ( $5 \mathrm{~ms}$ rise and fall times, cosine squared gated, $25 \mathrm{~ms}$ total duration) in a pseudorandom order within a double-walled sound chamber. Tonal signals were digitally generated with a 24 bit D/A converter at $156 \mathrm{kHz}$ (Tucker-Davis Technologies) and presented in the free-field $15 \mathrm{~cm}$ away from the left ear, measured at the center of the head. The stimuli set consisted of 16 intensities ranging from 0 to $75 \mathrm{~dB}$ sound pressure level (SPL) in $5 \mathrm{~dB}$ steps, and 129 frequencies in $1 / 16$ octave steps ranging from $250 \mathrm{~Hz}$ to $64,000 \mathrm{~Hz}$. Each frequency-intensity combination was presented once at a rate of $2.5 \mathrm{~Hz}$.

Recording procedures. Parylene-coated tungsten microelectrodes with impedances of $1-2 \mathrm{M} \Omega$ (FHC) were lowered $\sim 1200 \mu \mathrm{m}$ into auditory cortex. Microelectrode penetrations were limited to the gyral surface to decrease the likelihood of inconsistent laminae recording. Signals were bandpass filtered $(500 \mathrm{~Hz}$ to $5000 \mathrm{~Hz})$, amplified $(10,000 \times)$, and digitized at 25,000 Hz. Receptive fields were derived at distinct locations of auditory cortex to delineate the borders of A1 and A2 based on neuronal response properties (Merzenich et al., 1975; Knight, 1977; Reale and Imig, 1980; Schreiner and Cynader, 1984; Eggermont, 1998; Imaizumi et al., 2004). After demarcation of A1 and A2 borders was completed, a

\section{$\leftarrow$}

posterior ectosylvian sulcus and anterior ectosylvian sulcus are highlighted with black lines. Abbreviations: VAF, Ventral auditory field; FAES, auditory field of the anterior ectosylvian sulcus; $\mathrm{dPE}$, dorsal posterior ectosylvian area; $\mathrm{VPE}$, ventral posterior ectosylvian area; $\mathrm{PEE}$, intermediate posterior ectosylvian area. The sulci are indicated by italics. D, Dorsal; $A$, anterior; $P$, posterior; $V$, ventral. 
cooling loop was apposed to the midfrequency bands of A1 in four animals. Similarly, in the remaining four animals, a cooling loop was apposed to the center region of AII (Reale and Imig, 1980; Phillips and Orman, 1984). Location and size of cooling loops minimized the possibility of deactivation to adjacent cortical fields. An assessment of neuronal deactivation was conducted immediately after cooling loop placement. An electrode was lowered through the center of the cooling probe into layers IV/V and receptive fields were generated before, during, and after a period of cooling deactivation. Consequently, in four animals, receptive fields were derived in regions of A1 during epochs of A2 deactivation, whereas in the remaining four animals receptive fields were obtained from regions of A2 during stages of A1 cooling deactivation. During each recording cycle, receptive fields were generated at each of the five distinct thermal phases of cooling deactivation: before, transitioning to, during, transitioning out of, and after cooling. The neuronal activity thresholds were defined before the start of a recording cycle and were preserved until the completion of the sequence. The cooling extent was assessed by measuring the changes of cortical temperature at the end of each experiment by inserting a $0.2 \mathrm{~mm}$ outer diameter minihypodermic probe type $\mathrm{T}$ thermocouple (HYP-O; Omega) during a period of cooling deactivation to the same locations of previously recorded neuronal activity. A map of cortical temperature was produced to demarcate the extent of deactivation.

Reversible cooling deactivation. Various cooling loops were fabricated before each experiment from 23 gauge hypodermic tubing (Lomber et al., 1999). After the demarcation of the borders of A1 and A2 was completed, an appropriate cooling loop based on the size and shape of the required field was chosen. A copper/constantin microthermistor was attached at the union of the loop. Different cortical temperatures were achieved by pumping chilled methanol through the lumen of the cooling loop at various velocities. A wireless thermometer (UWTC-2; Omega) was used to monitor the temperature at the microthermistor. As previously shown (Lomber et al., 1994, 1999; Lomber, 1999; Chafee and Goldman-Rakic, 2000; Palmer et al., 2007; Nakamoto et al., 2008), neuronal activity of all cortical layers beneath the cooling loop was eliminated by maintaining the cooling probe at $3^{\circ} \mathrm{C}$.

Data analysis. All recorded receptive fields for the eight animals used in this study were randomized and analyzed by an experienced blind observer. Receptive fields were quantified based on characteristic frequency, neuronal threshold, and receptive field bandwidths. Characteristic frequencies were defined as the tone frequency that evoked a reliable response at the lowest intensity level; neuronal thresholds were determined to be the minimum intensity level (in decibels SPL) that evoked a consistent neural response; last, receptive field bandwidths were obtained by measuring the tuning curve width in octaves at $5 \mathrm{~dB}$ SPL steps above neuronal threshold. Peristimulus time histograms (PSTHs) from all the responses to tonal stimulation at each recorded site were generated using a MatLab (Mathworks) custom program. The neuronal response strength was calculated as the maximum number of spikes per second within the PSTH and the noise level was defined as the mean spike rate $50 \mathrm{~ms}$ before tone presentation. The onset latency of a recording site was calculated as the elapsed time after acoustic stimulation in which neuronal activity reached 2 SDs above noise; the peak latency was defined as the elapsed

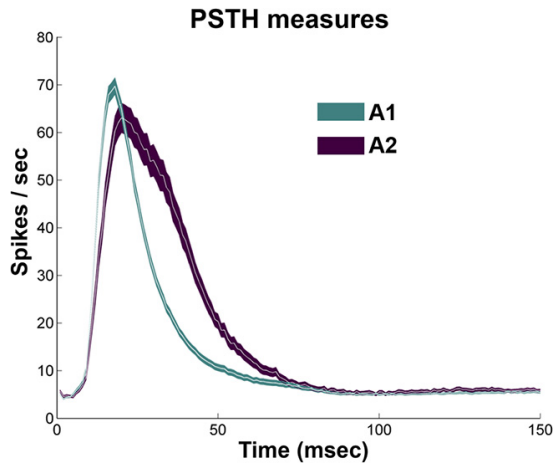

Figure 2. Population differences between $\mathrm{A} 1$ and $\mathrm{A} 2$ neurons in time course and duration of activity. PSTHs present the response activity of $1093 \mathrm{~A} 1$ sites and $260 \mathrm{~A} 2$ sites in response to tonal stimuli. The color region (purple and green) represents \pm SEM.

\section{A Characteristic frequency map}

\section{C}

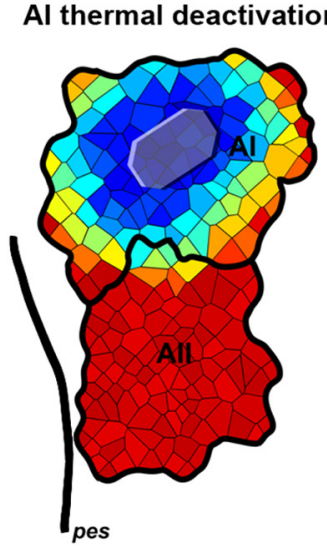

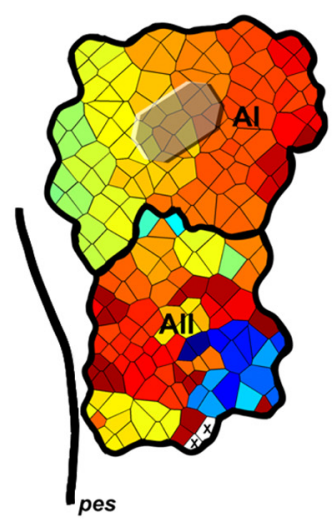

B Characteristic frequency map

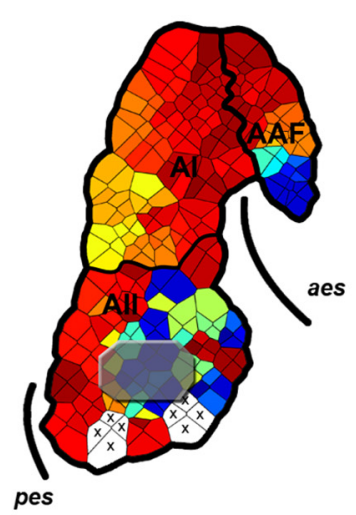

D All thermal deactivation

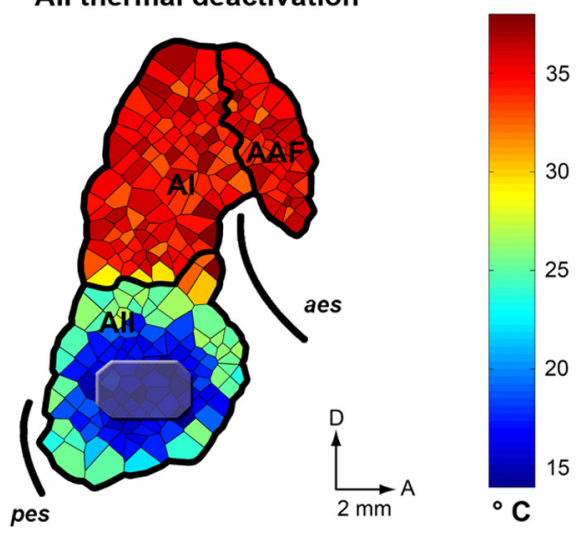

Figure 3. Representative examples of changes in cortical temperature during epochs of cooling deactivation. $A$, Characteristic frequency representation of primary and nonprimary fields of auditory cortex and representative location of A1 cooling loop (gray shaded region). $\boldsymbol{B}$, Characteristic frequency representation of primary and nonprimary fields of auditory cortex and representative location of A2 cooling loop (gray shaded region). C, Cortical temperature during periods of A1 cooling. D, Cortical temperature during periods of A2 cooling. Note that the cortical cooling of A1 or A2 regions did not extend into adjacent fields. The sulci are indicated by thick nonconnected black lines. X, Unresponsive cortical site. Abbreviations are as in Figure 1.

time after acoustic stimulation in which the maximum number of spikes occurred; and the end latency was measured as the elapsed time after acoustic stimulation in which evoked activity returned to within 2 SDs of noise level. The possibility that the changes observed were a consequence of neuronal death rather than cortical deactivation was reduced by excluding from additional analysis records of neuronal activity that did not return to at least $70 \%$ of their original firing rate during the rewarm period. Characteristic frequency and thermal cortical maps were constructed by generating Voronoi tessellations (Fig. 1C) (Kilgard and Mer- 


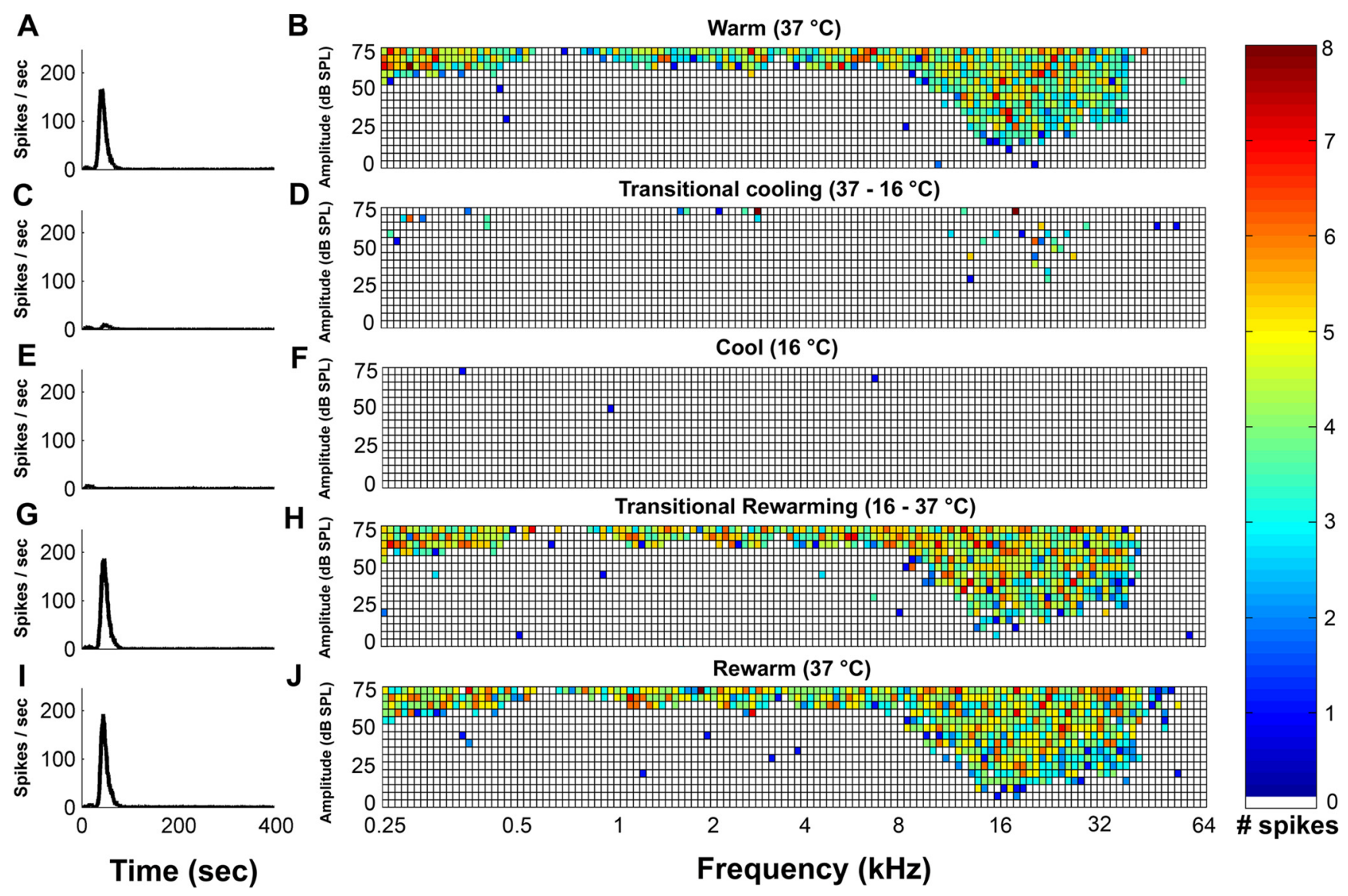

Figure 4. PSTHs and corresponding receptive fields of an A2 site recorded directly beneath a cryoloop during the five stages of a cooling cycle. Data were collected (1) before the initiation of cooling $(\boldsymbol{A}, \boldsymbol{B}),(2)$ during transitional cooling $(\boldsymbol{C}, \boldsymbol{D}),(3)$ while cooled $(\boldsymbol{E}, \boldsymbol{F}),(4)$ during transitional rewarming $(\boldsymbol{G}, \boldsymbol{H})$, and $(5)$ during rewarmed epochs $(\boldsymbol{I}, \boldsymbol{J})$. Note that each square in the grid represents a frequency-intensity combination presented during the recording period. The white squares indicate no spikes during the recording period in response to a tone presentation. Temperatures were measured at the cooling loop thermocouple and indicate the stage of the cooling cycle.

zenich, 1998). A two-tailed unpaired Student $t$ test was used for all statistical comparisons in this study. The description of Al response characteristics was accomplished by combining Al recordings conducted in this investigation with previously published A1 response properties of eight separate animals (Carrasco and Lomber, 2009a,b).

Histology. In two animals, an assessment of the cortical layer from which microelectrode recordings were made was conducted. Cats were perfused through the ascending aorta, with $0.1 \mathrm{~m}$ PBS for $5 \mathrm{~min}$ while the arterial system was infused with aldehyde fixatives (1.5\% gluteraldehyde $/ 1 \%$ paraformaldehyde in 0.1 M PBS) for $20 \mathrm{~min}$. Subsequently, $10 \%$ sucrose in $0.1 \mathrm{M}$ PBS was perfused for $5 \mathrm{~min}$. The solutions were infused at a rate of $100 \mathrm{ml} / \mathrm{min}$. Brains were photographed and placed in $30 \%$ sucrose in $0.1 \mathrm{M}$ PBS until they sank. Coronal sections ( $50 \mu \mathrm{m}$ thick) were cut and collected serially. Every fifth section was processed histochemically for the presence of cytochrome as we have done in the past (Payne and Lomber, 1996), and adjacent sections were stained using conventional methods for the presence of Nissl bodies.

\section{Results}

The objective of this investigation was to assess the bidirectional functional contributions between $\mathrm{A} 1$ and $\mathrm{A} 2$ of the cat. The results are presented in six sections. First, general response characteristics of A1 and A2 neurons are described and compared. Second, effects of cortical cooling on neuronal activity are illustrated. Third, changes in response properties of A1 neurons during A2 cooling deactivation are examined. Fourth, changes in response properties of A2 neurons during periods of A1 cooling deactivation are reported. Fifth, effects of cooling deactivation on neurons tuned to the deactivated region of A1 or A2 are pre- sented. Last, the relationship between response latency and magnitude of response change is examined.

\section{Comparison of $\mathrm{A} 1$ and $\mathrm{A} 2$ response properties \\ Location}

Neuronal response characteristics were used to determine the borders of A1 and A2 in each experiment. A lack of tonotopy in the dorsal zone of auditory cortex (DZ) and A2 was used as an indicator of the dorsal and ventral limits to A1, respectively. In comparison, reversals in tonotopic representation anteriorly and posteriorly of A1 were used as border demarcations between A1 and the anterior auditory field (AAF), as well as A1 and the posterior auditory field (PAF) (Fig. $1 A$ ). In addition to variations in tonotopicity, the characteristically long response latencies of PAF neurons and fast responses of AAF neurons were used as markers of A1 borders. Similarly, tonotopicity and latency measures were used to delineate $\mathrm{A} 2$ borders.

\section{Tuning representation}

All A1 cortices studied were found to be tonotopically organized. Neurons tuned to low frequencies were located on the banks of PES and a gradual increase in frequency tuning was observed in a posterio-anterior direction, culminating near the anterior ectosylvian sulcus (AES), where reversal in tuning organization marked the border with AAF (Fig. 1C). In contrast, A2 cortices did not display a consistent discernable tuning organization. 


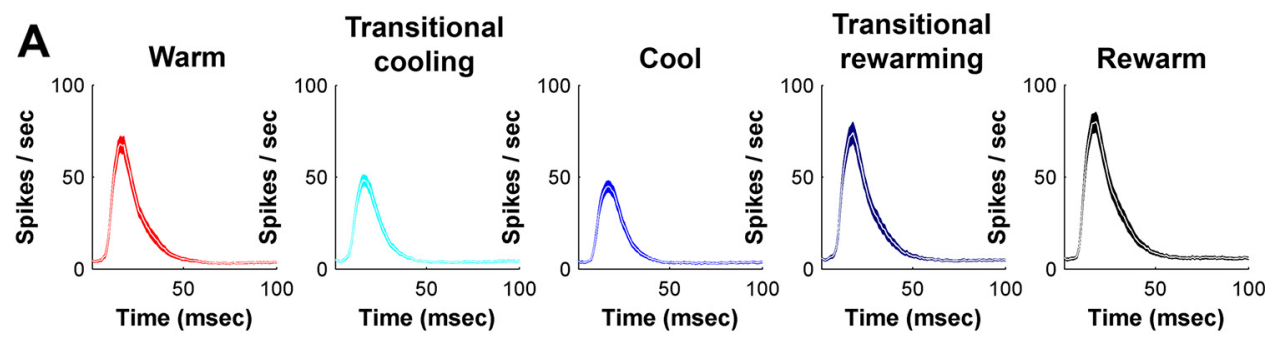

B
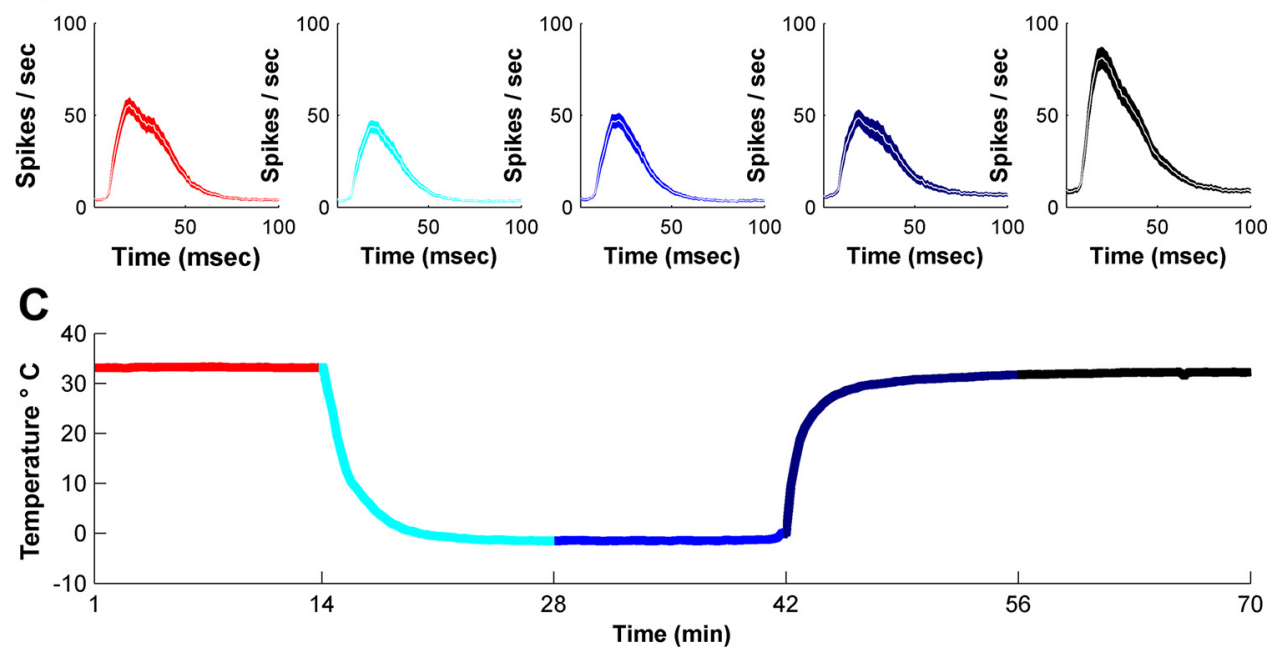

Figure 5. A, A1 neuronal activity during the five stages of $A 2$ cooling deactivation. $B, A 2$ neuronal activity during the five stages of $A 1$ cooling deactivation. $C$, Average temperature changes recorded at the cooling loop during A1 and A2 deactivation. The colors of the panels in $\boldsymbol{A}$ and $\boldsymbol{B}$ match the colors of $\boldsymbol{C}$ and indicate the times during which the PSTHs were constructed. Note the substantial decrease in A1 activity during A2 deactivation.

Temporal measures

The onset, peak, and end response latency measures to tonal stimulation of $1093 \mathrm{~A} 1$ and 260 A2 cortical locations were quantified. Although the onset response properties of A1 and A2 neurons did not exhibit substantial differences, comparisons of the peak and end latency measures revealed significantly longer A2 responses $(p<0.000005$; A1 mean peak response, $21.60 \mathrm{~ms} ; \mathrm{A} 2$ mean peak response, 23.98 $\mathrm{ms} ; p<0.000001$; A1 mean end response, $41.26 \mathrm{~ms}$; A2 mean end response, 50.29 $\mathrm{ms})$. The differences in evoked neuronal response properties between $\mathrm{A} 1$ and $\mathrm{A} 2$ neurons provide evidence that acoustic information arrives in both fields simultaneously but is subsequently processed at different timescales, with A2 neurons displaying a sustained response that results in longer peak and end latency measures than A1 neurons (Fig. 2).

\section{Effects of deactivation on}

\section{neuronal response}

The conclusions of this investigation are based on the effects of cooling on neuronal activity. Consequently, a meticulous assessment of these effects was conducted during each experiment. First, the area of
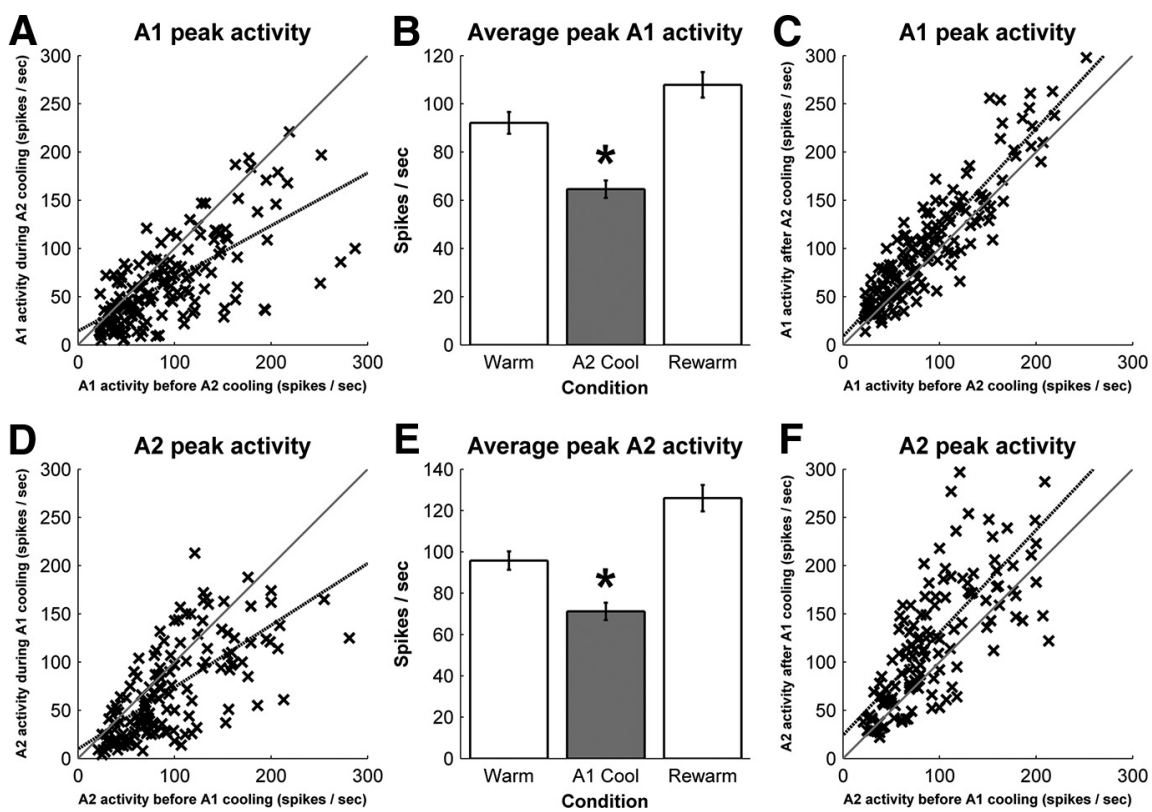

Figure 6. Mean peak response strength to tonal stimuli before, during, and after cooling deactivation. $A$, Response of $A 1$ neurons before ( $x$-axis) and during ( $y$-axis) A2 cooling. Similarly plotted is the peak response strength of $A 2$ neurons during $A 1$ cooling $(\boldsymbol{D})$. Average peak response strength of $A 1(\boldsymbol{B})$ and $A 2(\boldsymbol{E})$ neurons recorded before, during, and after periods of cooling deactivation. $\boldsymbol{C}$, Response of A1 neurons before ( $x$-axis) and after ( $y$-axis) A2 deactivation. $\boldsymbol{F}$, Response of $\mathrm{A} 2$ neurons before ( $x$-axis) and after ( $y$-axis) $\mathrm{A} 1$ deactivation. Note that both conditions result in a statistically significant reduction of neuronal firing. Error bars indicate \pm SEM. $A 1, n=156 ; A 2, n=139$. Statistical significance is at ${ }^{*} p<0.00007$. 

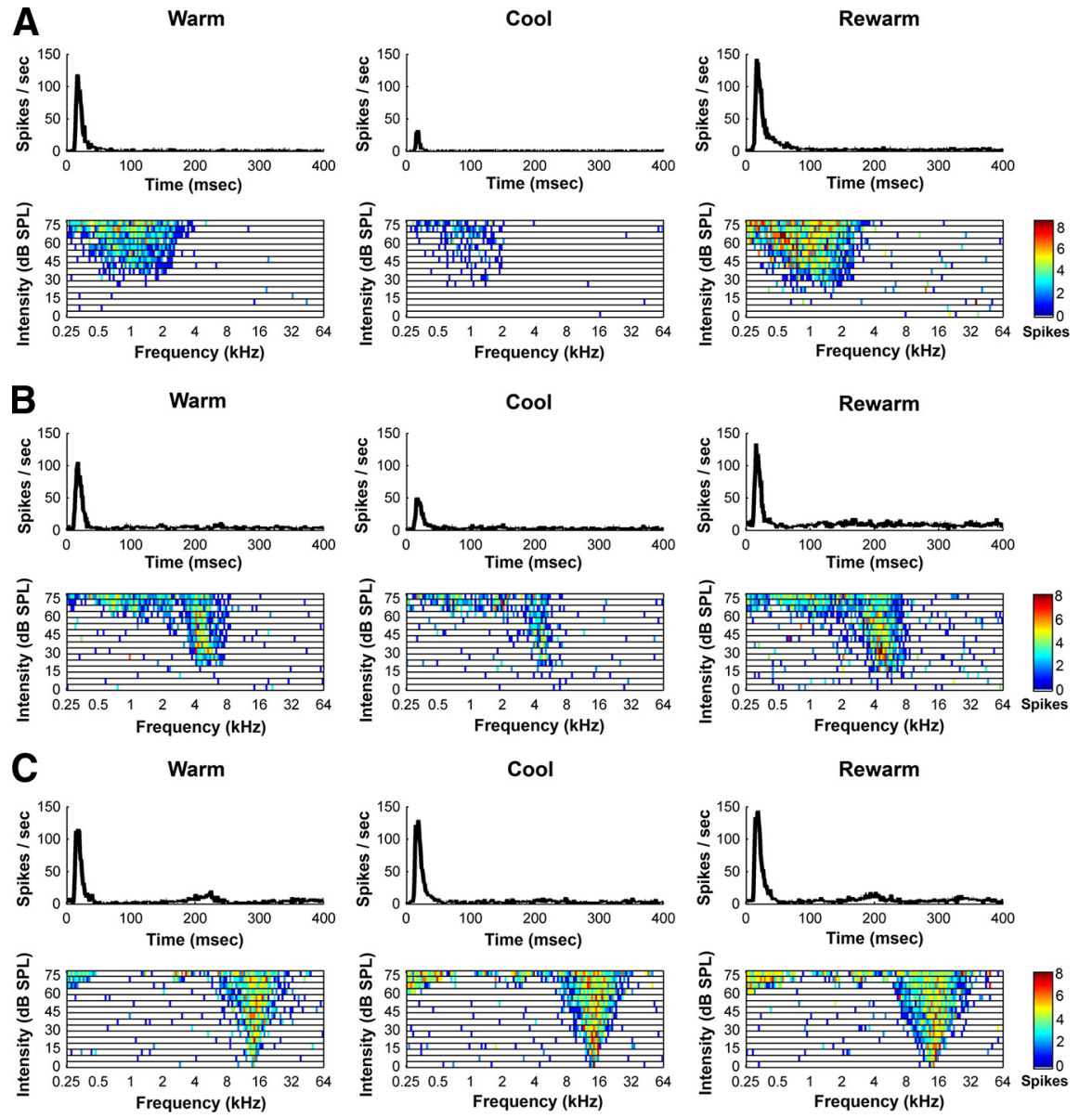

Figure 7. Representative changes in acoustically evoked activity of A1 neurons before, during, and after the cooling deactivation of A2. Three main types of changes were observed in A1 receptive fields and PSTHs during the cooling deactivation of A2 as follows: large effects incurred by $A 1$ neurons during $A 2$ deactivation $(\boldsymbol{A})$; midsize decrease in $A 1$ neuronal activity during $A 2$ deactivation $(\boldsymbol{B})$; and small response change of $A 1$ neurons during the deactivation of $A 2$ neurons $(\boldsymbol{C})$.

interest (A1 or A2) was identified by mapping neuronal response properties to tonal stimuli. After the borders of the region of interest were defined, a cooling loop was apposed to the center of the field (Fig. $3 A, B$ ). This arrangement minimized the possibility of cooling deactivation outside the perimeter of the field and maximized the amount of surface deactivation within the cortical region. Although the likelihood of cooling adjacent regions was low, we verified that no cooling contamination was present during neuronal recordings by measuring the extent of cortical cooling at the end of each experiment. The area of deactivation was revealed by generating cortical thermal maps during epochs of $\mathrm{A} 1$ or A2 cooling deactivation (Fig. 3C,D). As illustrated in Figure 3, $C$ and $D$, deactivation of $A 1$ or $A 2$ was contained within the loci of interest and did not extend into bordering cortical fields. Last, the magnitude of neuronal deactivation was assessed by measuring the changes in activity during epochs of cortical cooling at a site immediately underneath the cooling loop placement. Receptive fields (Fig. 4, right) and PSTHs (Fig. 4, left) were generated during each of the five stages of a single cooling cycle. It is evident from this analysis that neuronal activity before and after deactivation did not vary extensively, but the response activity during cooling deactivation was entirely abolished.

For each of the deactivated cortical sites (A1 or A2), the cytoarchitecture of Nissl-stained sections was characteristic of healthy cortex (Rose, 1949; Sousa-Pinto, 1973; Kelly and Wong, 1981). We were unable to find evidence of physical damage, gliosis, or necrosis. In addition, no changes were identified in cytochrome oxidase histochemistry. Therefore, in agreement with previous studies, neither the presence of the cryoloops nor their repeated deactivation changed the structure or long-term function of the two cortical areas assayed (Yang et al., 2006). Histological examination revealed that the overwhelming majority of recording sites were in layers IV and V, with lesser numbers of recordings sites in layers III and VI.

\section{Effects of A2 deactivation on A1 response properties}

Cooling deactivation of A2 neurons provoked variations in spectral and temporal response characteristics of Al cells. Specifically, these changes included reductions in response strength, increases in neuronal threshold, and decreases in response latency measures. In contrast, no significant changes were observed in receptive field bandwidths.

\section{Response strength}

The magnitude of acoustically evoked activity of A1 neurons before, during, and after A2 cooling deactivation was measured. Cumulative group PSTHs were generated for each of the five stages of the recording cycle (Fig. 5A,C). This approach revealed a significant decrease $(p=0.000014)$ of $34.65 \%$ in peak response strength during periods of A2 cooling deactivation. Although this analysis demonstrated a general decrease in response strength, the innate variability in peak response timing among A1 neurons resulted in an underestimation of the changes in response strength magnitude. To circumvent this undesirable effect, we confirmed these results with a second analysis that calculated the maximum number of spikes evoked during each stage of a recording cycle. The difference in A1 peak response strength before, during, and after A2 deactivation was calculated (Fig. $6 A-C)$. A significant difference $(p<$ $0.000003)$ in the average of A1-driven responses before and during epochs of A2 cooling deactivation was revealed (Fig. $6 B)$. This quantification approach showed a reduction in response strength in the majority of A1 neurons. Specifically, in 23 of 156 A1 sites examined, a decrease of $66 \%$ or more of the original firing rate was recorded during epochs of A2 deactivation (Fig. 7A); in 42 of 156 sites, a reduction between 33 and $66 \%$ of the original firing rate was measured (Fig. $7 B$ ); and in 57 of 156 recorded sites, a change of $<33 \%$ from the original firing rate was revealed. In contrast, the remaining 34 cells showed a small increase in peak response (Fig. 7C). The two analytical approaches implemented provide evidence of a modulatory influence of A2 neurons over A1 acoustically driven responses. 
Neuronal threshold

We measured the neuronal sensitivity of A1 sites by identifying the minimum intensity (in decibels SPL) needed to produce a reliable neuronal response. A comparison between sensitivity levels before, during, and after A2 deactivation revealed a significant increase $(p=0.0026)$ of $2.7 \mathrm{~dB}$ during cooling epochs. It was then followed by a return to baseline levels during the rewarm condition. Figure $8 A-C$ illustrates the individual and group neuronal threshold variations of A1 cells during A2 cooling deactivation. The observed changes in neuronal thresholds suggest that A2 may be involved in the modulation of A1 sensitivity levels to tonal signals.

\section{Response latencies}

Onset, peak, and end A1 latency response times were measured before, during, and after epochs of A2 deactivation. Figure $9 A-C$ illustrates the response timing variations of A1 neurons before and during periods of A2 deactivation. Although no changes were observed in the onset and peak latency responses of A1 neurons, a significant decrease in end latency ( $p=$ 0.0044; before, $35.7 \mathrm{~ms}$; during, $31.97 \mathrm{~ms}$ ) was revealed (Fig. 9D). This analysis suggests that only the latter part of A1 neuronal responses may be affected by the deactivation of $\mathrm{A} 2$ neurons.

\section{Receptive field bandwidth}

The potential modulatory effect of A2 neurons on $\mathrm{A} 1$ receptive field bandwidths was analyzed. Changes in bandwidths were measured in increments of $5 \mathrm{~dB}$ steps from the neuronal threshold. Statistically significant changes $(p<0.0373)$ were only observed at $50 \mathrm{~dB}$ SPL above neuronal threshold. The lack of consistent bandwidth variation implies that certain aspects of A1 auditory receptive field properties may not be regulated by A2 activity.

\section{Effects of A1 deactivation on A2 response properties}

Analogous measures to the aforementioned quantification techniques were conducted to identify the effects of A1 neuronal deactivation on the response properties of A2 neurons. The data analyses revealed variations in response strength, neuronal threshold, and latency measures. In contrast, no consistent changes in receptive field bandwidths were identified.

\section{Response strength}

Contradictory to the response changes observed in A1 neurons during A2 cooling deactivation, no statistically significant reduction in the response magnitude of A2 neurons was identified during epochs of A1 deactivation (Fig. 5B). This result is not surprising as A2 neurons exhibit a large variability in peak response latency resulting in the underestimation of changes in response magnitude. This technical problem was evaded by measuring the peak activity of each A2 neuronal recording before, during, and after A1 deactivation. These measures allowed us to make direct comparisons of response strength variations for each collected record. The assessment of the observed changes is pre-
B

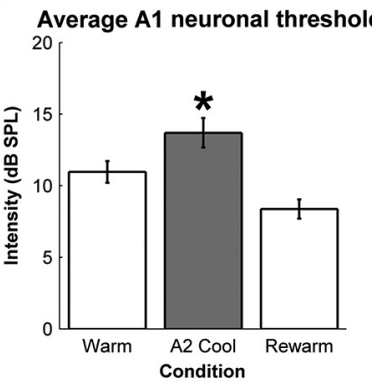

C

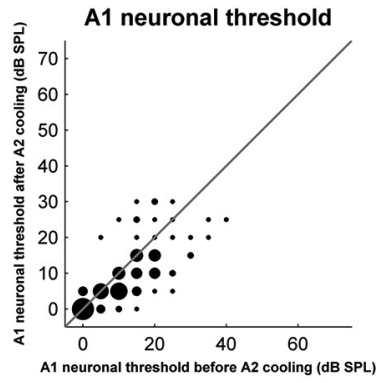

E

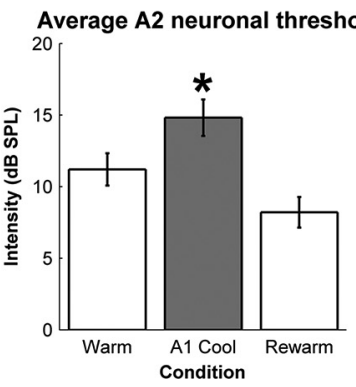

F

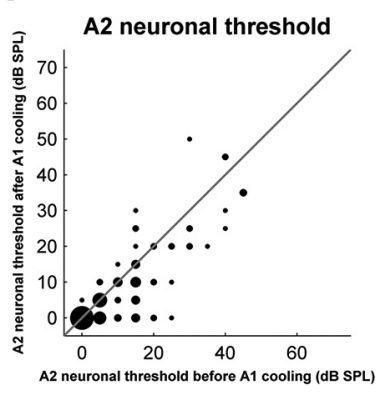

Figure 8. Neuronal thresholds before, during, and after cooling deactivation. $A$, A1 neuronal threshold measures before ( $x$-axis) and Average threshold measures of $A 1(B)$ and $A 2(\boldsymbol{E})$ neurons recorded before, during, and after periods of cooling deactivation. $C$, Thresholds both conditions. Error bars indicate \pm SEM. $A 1, n=139 ; A 2, n=112$. In $B$, statistical significance is at ${ }^{*} p=0.0026$. In $\boldsymbol{E}$, statistical significance is at ${ }^{*} p=0.034$. In $A$ and $\boldsymbol{C}$, circle sizes range from 1 to 28 occurrences. $\operatorname{In} \boldsymbol{D}$ and $\boldsymbol{F}$, circle sizes range from 1 to 24 occurrences.
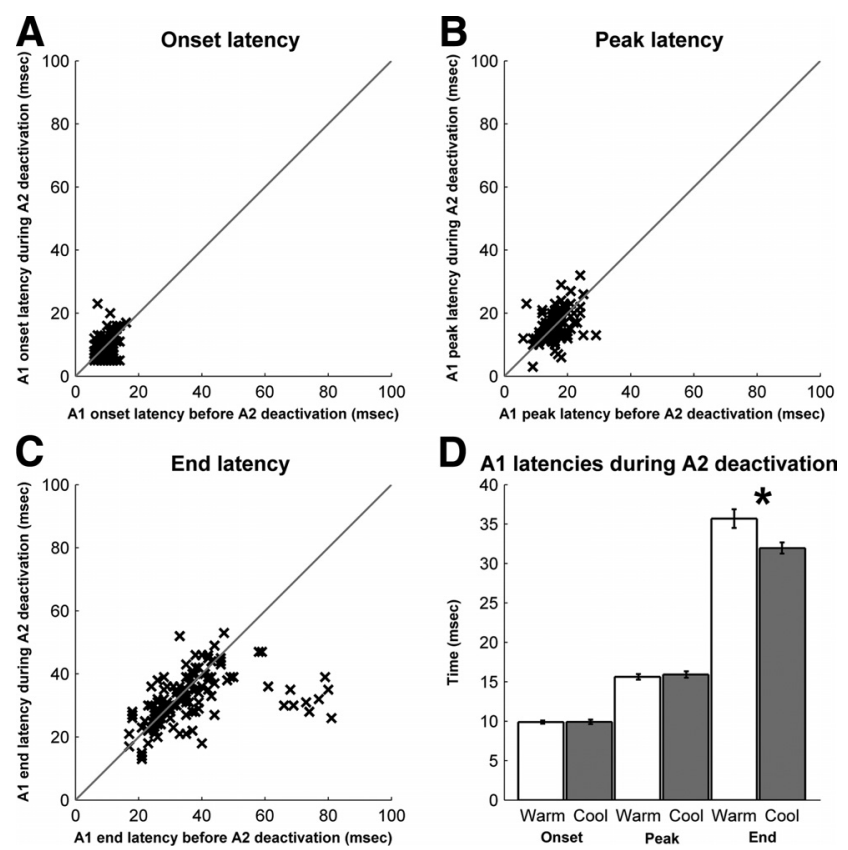

Figure 9. Neuronal latency changes in A1 sites during periods of $A 2$ cooling deactivation. $\boldsymbol{A}-\boldsymbol{C}$, Changes in onset latency $(\boldsymbol{A})$, peak latency $(\boldsymbol{B})$, and end latency $(\boldsymbol{C})$. A1 latency values are plotted before ( $x$-axis) and during ( $y$-axis) A2 deactivation for each latency measure. $D$, Summary of mean neuronal changes in A1 latency measures during $A 2$ deactivation. Errorbars indicate $\pm S E M ; n=139$. Statistical significance is at ${ }^{*} p=0.0044$.

sented in Figure 6D-F. Statistically significant reductions in A2 neuronal response magnitude levels $(p<0.00007)$ were identified during, but not after, periods of A1 deactivation (Fig. 6E). This analysis revealed that, in 22 of 139 A2 sites examined, a 

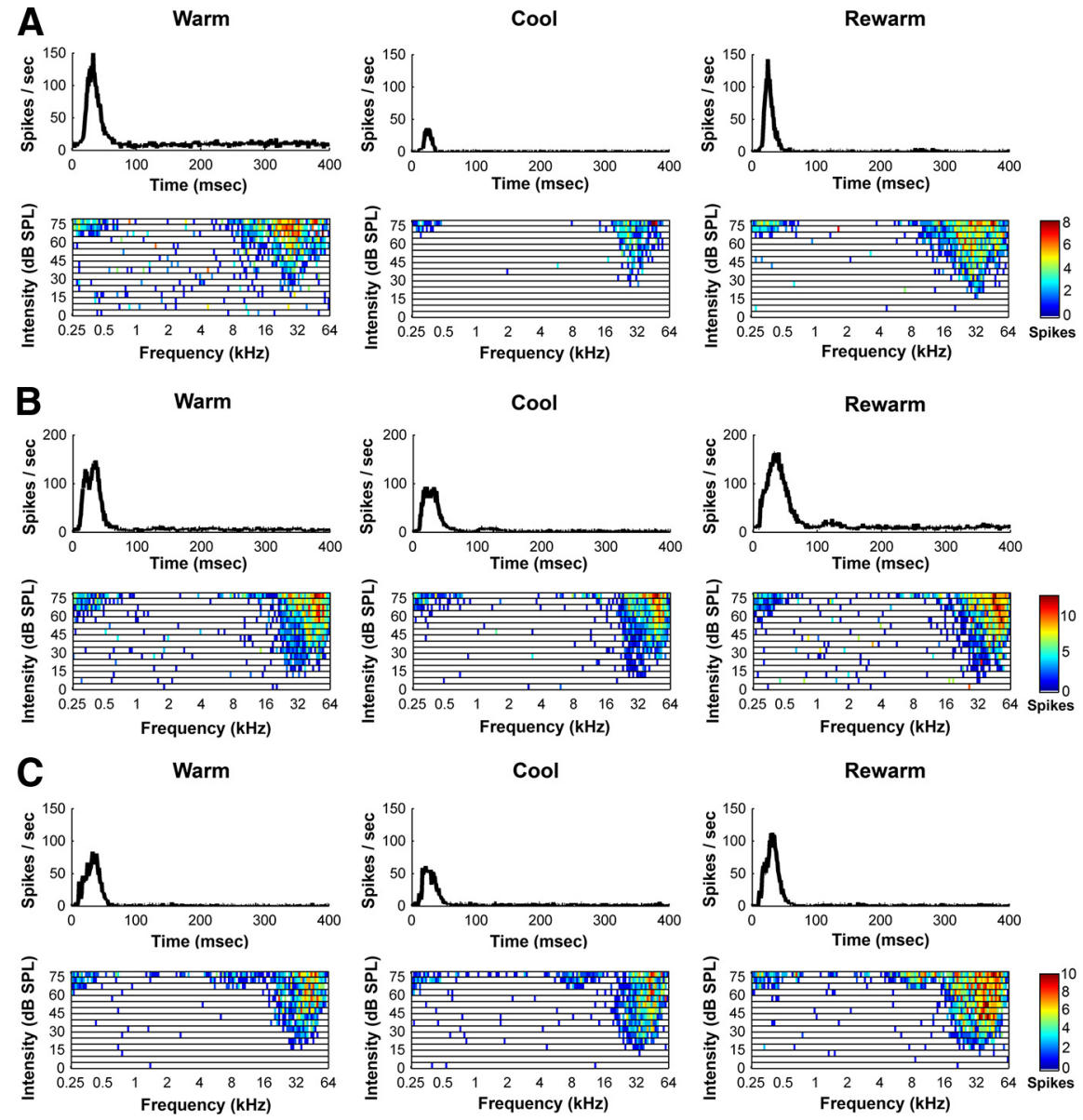

Figure 10. Representative changes in acoustically evoked activity of A2 neurons before, during, and after the cooling deactivation of A1. Three main types of changes were observed in $A 2$ receptive fields and PSTHs during the cooling deactivation of $A 1$ as follows: large effects incurred by $A 2$ neurons during $A 1$ deactivation $(\boldsymbol{A})$; midsize reduction of $A 2$ neuronal activity during $A 1$ deactivation (B); and small response change of $A 2$ neurons during the deactivation of $A 1$ neurons $(\boldsymbol{C})$.

decrease of $66 \%$ or more of the original firing rate was recorded during epochs of A1 deactivation (Fig. 10A); in 47 of 139 sites, a reduction between 33 and $66 \%$ of the original firing rate was measured (Fig. $10 \mathrm{~B}$ ); and in 36 of 139 recorded sites, a change of $<33 \%$ from the initial firing rate was observed. In the remaining 34 cells, a small increase in peak response was measured (Fig. $10 C)$. Therefore, this analysis revealed a substantial response decrease of A2 activity during epochs of A1 deactivation.

\section{Neuronal threshold}

Similarly to the changes observed in A1 cells during A2 deactivation, the silencing of A1 neuronal activity provoked significant increases $(p=0.034)$ in A2 neuronal thresholds. Before periods of A1 deactivation, the average threshold of A2 neurons measured $11.21( \pm 0.79) \mathrm{dB}$ SPL. In contrast, this level increased to $14.82( \pm 0.89) \mathrm{dB}$ SPL during epochs of A1 deactivation (Fig. $8 D-F)$. The observed changes provide evidence that A2 functional properties can be influenced by A1 activity.

\section{Response latencies}

Onset, peak, and end latencies of A2 cells before, during, and after the deactivation of A1 activity were calculated. This analysis revealed statistically significant decreases in peak $(p<0.01)$ and end $(p<0.0001)$ response latencies during epoch of A1 deactivation (Fig. $11 A-D$ ). No change in response onset was identified, indicating that only the late response time of $\mathrm{A} 2$ cells can be regulated by $\mathrm{A} 1$ activity.

\section{Bandwidth}

Receptive field bandwidths were calculated for A2 neurons before, during, and after A1 neuronal deactivation. In general, a monotonic increase in bandwidth measures was revealed as a function of intensity. Significant differences $(p<0.012)$ were found only at $5 \mathrm{~dB}$ SPL above neuronal threshold. The lack of change observed during deactivation epochs implies that A1 does not have a large impact on A2 receptive field bandwidths.

\section{Effects of tuning properties on neuronal response changes}

Variations in tuning properties during the period of cooling deactivation were investigated. The goal of this analysis was to determine the involvement of each field on the tuning characteristics of the other. Neither A1 nor A2 neurons exhibited statistically significant changes in characteristic frequency during periods of deactivation (Fig. 12). In a second analysis, the cortical frequency tuning maps generated during the first stage of each experiment in combination with the thermal maps created at the end of each procedure, allowed us to identify the tuning features of neurons that were affected by cortical cooling (Fig. 3). In general, A1 deactivation encompassed the frequency range from $2000 \mathrm{~Hz}$ to $32,000 \mathrm{~Hz}$. In comparison, the lack of tonotopic organization of A2 neurons resulted in the deactivation of clusters of various frequency ranges (Fig. 3B). For this analysis, the peak response strength magnitude of each recorded site was grouped based on characteristic frequency in one of three frequency bins. A1 neurons tuned to frequencies from 16,000 to $64,000 \mathrm{~Hz}$ exhibited a significantly less pronounced decrease in response magnitude than neurons with characteristic frequencies from 1000 to $4000 \mathrm{~Hz}$ ( $p=0.012$ ), and neurons with characteristic frequencies ranging from 4000 to $16,000 \mathrm{~Hz}(p<$ 0.0000002 ) (Fig. 13A). In comparison, no significant variations in response magnitude as a function of tuning properties was observed in A2 neurons during A1 deactivation (Fig. 13B). The revealed changes provide evidence that specific regions of $A 1$ are affected differentially by A2 deactivation and that this effect is not transferable to A2 neurons, in which a similar decrease in response magnitude was observed regardless of tuning properties.

\section{Effects of response latency properties on neuronal response changes}

Last, we quantified the effects of end latency measures on the observed variations of response magnitude. The aim of this analysis was to determine whether neurons with longer response properties undergo a more pronounced decrease in response strength than neurons with shorter response latencies. The difference in response times of A1 and A2 neurons was taken into account by grouping the data into distinct response time bins. A1 data were divided into three groups (bin 1, 10-20 ms; bin 2, 20-30 ms; bin 3, 30-40 ms). In comparison, the longer response 

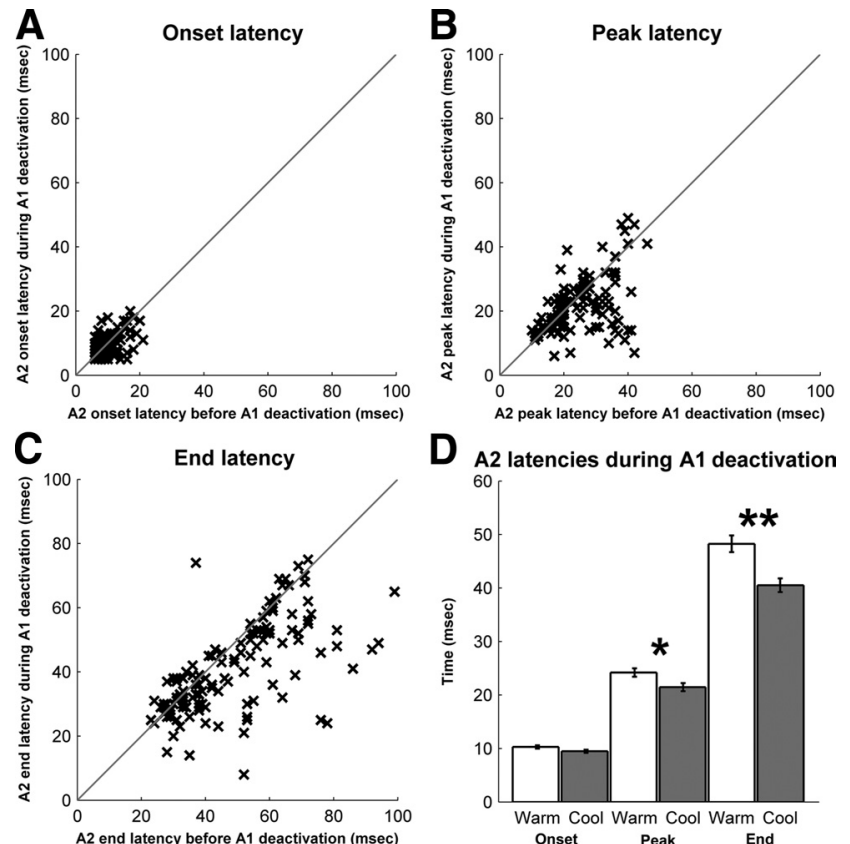

Figure 11. Neuronal latency changes in $A 2$ sites during periods of $A 1$ deactivation. $\boldsymbol{A}-\boldsymbol{C}$, Changes in onset latency $(\boldsymbol{A})$, peak latency $(\boldsymbol{B})$, and end latency ( $\boldsymbol{C}$. A2 latency values are plotted before ( $x$-axis) and during ( $y$-axis) A1 deactivation for each latency measure. $\boldsymbol{D}$, Summary of mean neuronal changes in A2 latency measures during A1 deactivation. Note that statistically significant differences were observed at the peak and end latency measures. Error bars indicate $\pm \mathrm{SEM} ; n=133$. Statistical significance is at ${ }^{*} p=0.0095$ and ${ }^{* *} p=0.00008$.

properties of A2 neurons was subdivided into groups with appropriate response time ranges (bin 1, 20-40 ms; bin 2, 40-60 ms; bin 3, 60-80 ms). The results of this analysis revealed that A1 neurons with short response properties $(<20 \mathrm{~ms})$ were significantly $(p<0.05)$ less affected during A2 deactivation than A1 neurons with longer response latencies (Fig. 14A). Similarly, A2 neurons with short latency measures $(<40 \mathrm{~ms})$ were significantly $(p=0.004)$ less affected than neurons with response latencies ranging from 40 to $60 \mathrm{~ms}$ (Fig. 14B). These results provide evidence that A1 neurons with short response latencies are less influenced by A2 deactivation than neurons with longer response times and that this effect is transferable to A2 neurons, in which cells with short latencies exhibited a small change in response strength during A1 deactivation.

\section{Results summary}

The objective of the present investigation was to study the reciprocal functional contributions between $\mathrm{A} 1$ and $\mathrm{A} 2$ of the cat. Changes in acoustically evoked neuronal responses were observed in a large number of A1 sites during the synaptic silencing of A2 neurons. Similarly, the response properties of a substantial number of A2 sites were affected by the cooling deactivation of A1 cells. Consequently, these findings provide evidence of a mutual modulatory interaction between A1 and A2 neurons. Furthermore, the results of the present study are in agreement with previous reports on the response properties of primary and nonprimary auditory field neurons. In particular, evidence of A1 tonotopic organization, as well as a lack of tonotopy in A2, was substantiated (Reale and Imig, 1980; Schreiner and Cynader, 1984).
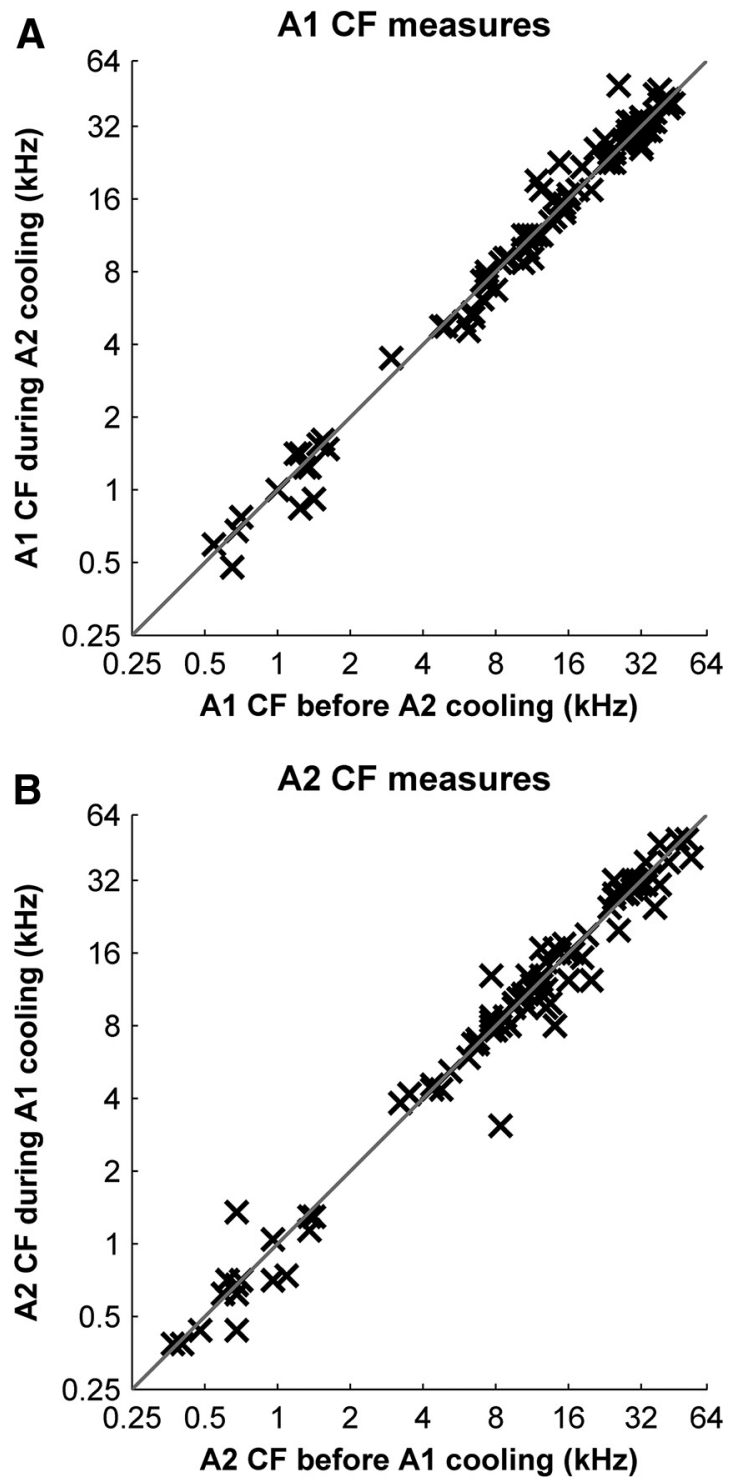

Figure 12. $A$, Characteristic frequencies (CFs) of $A 1$ neurons before and during cooling deactivation of $A 2$. $B$, CFs of $A 2$ neurons before and during cooling deactivation of A1. CFs are plotted in a before ( $x$-axis) and during ( $y$-axis) cooling deactivation manner. Note: No statistically significant changes were observed in CF shifts during the cooling deactivation of $A 1$ or $A 2$. $\mathrm{A} 1, n=139 ; \mathrm{A} 2, n=112$.

\section{Discussion}

Interactions in auditory cortex

In an attempt to further our understanding of sensory signal processing in cortex, the present study investigated the response characteristics and functional interactions between primary and nonprimary auditory fields in the cat. In addition to other reports (Rauschecker et al., 1997; Romanski et al., 1999; Eggermont, 2000; Carrasco and Lomber, 2009a,b; Kusmierek and Rauschecker, 2009), our results provide insight into basic rules of acoustic information processing in cortex. Specifically, this investigation illustrates interactions between primary and nonprimary fields of auditory cortex. Additionally, the present observations help elucidate the contribution that Al makes to information processing in higher order areas.

\section{Interfield corticocortical interactions}

Functional and anatomical studies in felines and primates have provided evidence of a hierarchical organization among the fields 

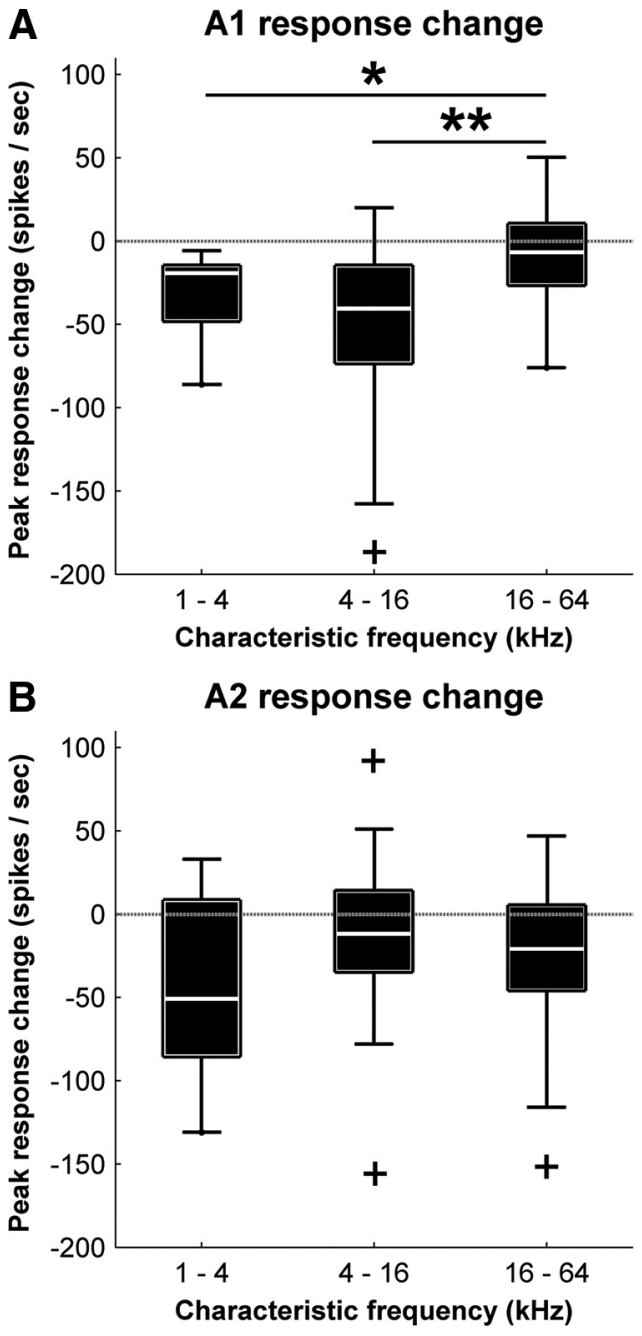

Figure 13. Variations in response activity based on characteristic frequency properties. $A$, Changes in $\mathrm{A} 1$ response activity during $\mathrm{A} 2$ cooling deactivation in neurons with receptive fields tuned to specific frequency ranges. $\boldsymbol{B}$, Changes in $\mathrm{A} 2$ response activity during $\mathrm{A} 1$ cooling deactivation in neurons with receptive fields tuned to low, middle, or high frequencies. The lines in the boxplots illustrate the lower quartile, median, and upper quartile values. The whiskers from each end of the boxes show the extent of the rest of the data, and outliers are shown with + signs beyond the ends of the whiskers. $A 1, n=132 ; A 2, n=112$. Statistical significance is at ${ }^{*} p=0.012$ and ${ }^{* *} p=0.0000002$.

that form auditory cortex. Specifically, functional studies in the macaque have shown a strong A1 influence on response properties of caudio-medial area neurons but not on neurons in area $\mathrm{R}$ (Rauschecker et al., 1997). Similarly, functional investigations in the cat have revealed a distinctive pattern of interactions between A1, AAF, and PAF (Carrasco and Lomber, 2009a,b). The findings of these studies suggest that interactions between cortical regions influence specific neuronal functional properties. The results of the present investigation support the existence of a functional organization among regions of auditory cortex by providing evidence that primary and nonprimary fields of auditory cortex have the ability to modulate the response properties of one another.

\section{Implications of A1 activity}

Functional and anatomical studies in primates, felines, and rodents have frequently reported the response properties and structural characteristics of A1 neurons (Evans et al., 1965; Abeles and Goldstein, 1970; Merzenich et al., 1973; Middlebrooks and
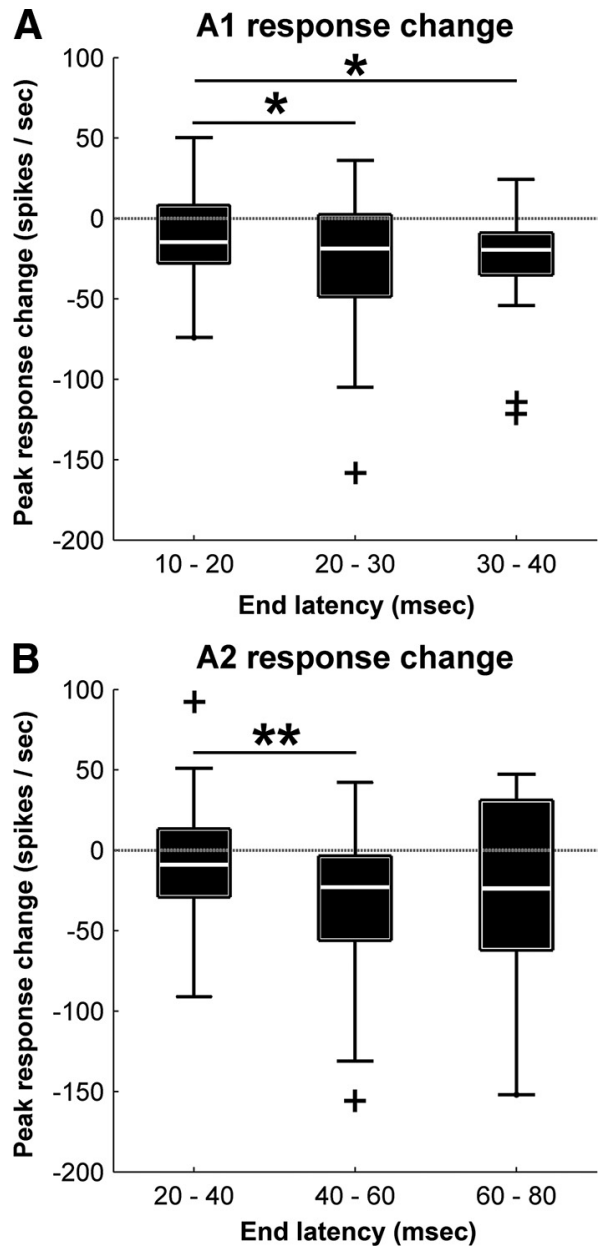

Figure 14. Variations in response activity based on end-latency measures. $A$, Changes in $\mathrm{A} 1$ response activity during $A 2$ cooling deactivation in neurons with specific response latencies. $B$, Changes in $A 2$ response activity during $A 1$ cooling deactivation in neurons with response properties lasting specific time intervals. The lines in the boxplots illustrate the lower quartile, median, and upper quartile values. The whiskers from each end of the boxes show the extent of the rest of the data, and outliers are shown with + signs beyond the ends of the whiskers. A1, $n=99 ; A 2, n=101$. Statistical significance is at ${ }^{*} p<0.05$ and ${ }^{* *} p=0.004$.

Pettigrew, 1981; Winguth and Winer, 1986; Kenet et al., 2007; Weinberger, 2007; Noreña et al., 2008) but have not revealed the functional communicative principles of this field. To identify the role of $\mathrm{A} 1$ on acoustic processing, it is imperative to understand its interactions with other cortical regions. A plausible postulate for its role in acoustic signal analysis places A1 as a relay distributor that coordinates the processing of sensory information. The results of the present investigation, in addition to previous reports, suggest that the response properties of A1 neurons not only depend on the activity of associative cortical fields but can substantially affect the response properties of nonprimary neurons (Rauschecker et al., 1997; Carrasco and Lomber, 2009a). The dependence and influence of A1 activity on nonprimary field neurons provide evidence of A1 neuronal involvement in multiple levels of analysis of auditory signals, thus supporting the existence of plausible parallel and sequential processing networks.

\section{Technical considerations}

Prevailing views in neurophysiology advocate the need to conduct studies like the present investigation in unanaesthetized preparations. Although it is crucial to acknowledge the construal limitation of the present results, certain aspects of this investiga- 
tion could not have been accomplished using an unanesthetized preparation. Specifically, fine cortical mapping, precise placement of cooling loops, and measures of deactivation spread are not feasible procedures in awake recordings. Thus, the present study should be considered as a necessary step in our understanding of intrafield communicative properties but should not be generalized to awake states. Nonetheless, expectations for comparable results between anesthetized and awake conditions can be based on visual cortex investigations. In particular, results of the effects of V1 deactivation on V2, V3, and V4, using similar preparations to the one utilized in the present study (Girard and Bullier, 1989; Girard et al., 1991a,b), have endured for nearly two decades. Only more investigations using both anesthetized and unanesthetized preparations will provide a clearer view of intrafield communication among regions of auditory cortex.

A second technical concern is the use of multiunit recordings. Although it is important to acknowledge the limitation of this technique, such as bandwidth and threshold measures in nontonotopic fields, the deactivation results of the present study are not affected by this drawback as each recording was conducted from the same group of cells before, during, and after epochs of deactivation. The use of multiunit recordings permitted the neuronal response measures of a wide cortical surface area while reflecting the integrated activity at each recorded location.

\section{Anatomical and functional models of cortical hierarchical structure}

Neuroanatomical studies have identified a dense network of connections among the fields that form auditory cortex (Rouiller et al., 1991; Hackett et al., 1998; Lee and Winer, 2008). In the cat, corticocortical connections have been labeled as feedforward, feedback, or lateral based on the origin and target layers of interfield projections (Rockland and Pandya, 1979; Felleman and Van Essen, 1991; Rouiller et al., 1991). Specifically, connections originating from superficial layers and terminating in layer IV have been categorized as ascending or feedforward. Conversely, projections originating from deep layers and terminating outside layer IV are generally referred to as descending or feedback. Last, projections originating from mixed cortical layers have been termed lateral or horizontal (Rouiller et al., 1991). Founded on the segregation and categorization of these three types of connections, schemes of serial and parallel cortical processing have been postulated (Rauschecker et al., 1997; Rauschecker, 1998). Although these anatomical models have demonstrated the existence of a well defined network of connectivity, the functional properties of each type of connection has not been investigated. Subsequently, we currently have a poor understanding of the functional differences between feedforward, feedback, and lateral connections in the auditory cortex. In addition, we do not have concrete evidence supporting a relationship between anatomical characteristics and flow of acoustic information in cortex.

It has been reported that the large anatomical differences in corticocortical connections in cat auditory cortex may not be the sole determinant of functional interaction between cortical fields (Carrasco and Lomber, 2009a,b). In the present study, anatomically defined feedforward connections $(\mathrm{A} 1 \rightarrow \mathrm{A} 2)$ (Rouiller et al., 1991) had a comparable functional role as the anatomically defined feedback projections (A2 $\rightarrow$ A1) (Rouiller et al., 1991). This result presents evidence that the distinct types of corticocortical projections connecting these two areas are similar in function. In comparison, the deactivation of Al on the response properties of PAF neurons, anatomically defined as feedforward projections (Rouiller et al., 1991), has been shown to have a strong influence on neuronal response magnitude. On the contrary, no large effects in A1 response properties during the deactivation of PAF neurons have been observed. These results indicate that the anatomical differences connecting A1 neurons and PAF neurons may exercise a vital role in interfield functionality. Last, the effects of deactivation of A1 activity on the response properties of neurons in the AAF, anatomically defined as lateral connections (Rouiller et al., 1991), are weaker than the effects observed in the reciprocal (lateral) connections from AAF to A1. In conclusion, functional reports in auditory cortex have not yet established a link between structure and function at the level of interfield connectivity. A possible source for this discrepancy may arise from the acoustic stimuli used in these experiments. In the aforementioned studies, simple acoustic stimuli were used, leaving the possibility that a distinct pattern of activity could result from complex acoustic signals. Evidence for this possibility has been shown in monkey studies in which simple and complex acoustic signals were presented after the physical removal of A1 (Rauschecker et al., 1997). In this study, it was shown that, although the response to simple stimuli was abolished in neurons of the caudal medial area, no significant deficit in response was observed when complex stimuli were presented. Therefore, future investigations should used simple, complex, and ecologically relevant stimuli to further our understanding of interfield interactions based on signal structure and behavioral significance.

\section{Converging inputs}

The findings of the present investigation have been interpreted as a consequence of direct corticocortical interactions; however, it is important to acknowledge the plausible influence of thalamocortico projections on the observed changes in neuronal activity. Specifically, deactivation of cortical neurons is likely to affect the response properties of thalamic cells via cortico-thalamic projections. Respectively, changes in thalamic activity present a potential indirect source of modulation to cortical neurons via thalamo-cortical connections. This type of cortico-thalamocortical loop has been proposed to be a fundamental property of visual information processing (Merabet et al., 1998; Cudeiro and Sillito, 2006; Sillito et al., 2006) and suggests a plausible form of auditory information processing. In the future, our understanding of how auditory cortex processes acoustic signals will benefit from investigations of the contributions of converging thalamocortical, transcallosal, and corticocortical inputs to the response properties of auditory neurons. It is the interaction of multiple cortical fields and not the isolated study of any one individual region that will truly advance our understanding of how sensory stimulation is processed by cortex.

\section{References}

Abeles M, Goldstein MH Jr (1970) Functional architecture in cat primary auditory cortex: columnar organization and organization according to depth. J Neurophysiol 33:172-187.

Amir Y, Harel M, Malach R (1993) Cortical hierarchy reflected in the organization of intrinsic connections in macaque monkey visual cortex. J Comp Neurol 334:19-46.

Bullier J (2001) Integrated model of visual processing. Brain Res Brain Res Rev 36:96-107.

Carrasco A, Lomber SG (2009a) Differential modulatory influences between primary auditory cortex and the anterior auditory field. J Neurosci 29:8350-8362.

Carrasco A, Lomber SG (2009b) Evidence for hierarchical processing in cat auditory cortex: nonreciprocal influence of primary auditory cortex on the posterior auditory field. J Neurosci 29:14323-14333.

Chafee MV, Goldman-Rakic PS (2000) Inactivation of parietal and prefron- 
tal cortex reveals interdependence of neural activity during memoryguided saccades. J Neurophysiol 83:1550-1566.

Cheung SW, Nagarajan SS, Bedenbaugh PH, Schreiner CE, Wang X, Wong A (2001) Auditory cortical neuron response differences under isoflurane versus pentobarbital anesthesia. Hear Res 156:115-127.

Coogan TA, Burkhalter A (1993) Hierarchical organization of areas in rat visual cortex. J Neurosci 13:3749-3772.

Cudeiro J, Sillito AM (2006) Looking back: corticothalamic feedback and early visual processing. Trends Neurosci 29:298-306.

Eggermont JJ (1998) Representation of spectral and temporal sound features in three cortical fields of the cat. Similarities outweigh differences. J Neurophysiol 80:2743-2764.

Eggermont JJ (2000) Sound-induced synchronization of neural activity between and within three auditory cortical areas. J Neurophysiol 83:27082722.

Evans EF, Ross HF, Whitfield IC (1965) The spatial distribution of unit characteristic frequency in the primary auditory cortex of the cat. J Physiol 179:238-247.

Felleman DJ, Van Essen DC (1991) Distributed hierarchical processing in the primate cerebral cortex. Cereb Cortex 1:1-47.

Girard P, Bullier J (1989) Visual activity in area V2 during reversible inactivation of area 17 in the macaque monkey. J Neurophysiol 62:1287-1302.

Girard P, Salin PA, Bullier J (1991a) Visual activity in macaque area V4 depends on area 17 input. Neuroreport 2:81-84.

Girard P, Salin PA, Bullier J (1991b) Visual activity in areas V3a and V3 during reversible inactivation of area $\mathrm{V} 1$ in the macaque monkey. J Neurophysiol 66:1493-1503.

Hackett TA, Stepniewska I, Kaas JH (1998) Subdivisions of auditory cortex and ipsilateral cortical connections of the parabelt auditory cortex in macaque monkeys. J Comp Neurol 394:475-495.

Imaizumi K, Priebe NJ, Crum PA, Bedenbaugh PH, Cheung SW, Schreiner CE (2004) Modular functional organization of cat anterior auditory field. J Neurophysiol 92:444-457.

Kaas JH, Hackett TA (1999) "What" and "where" processing in auditory cortex. Nat Neurosci 2:1045-1047.

Kelly JP, Wong D (1981) Laminar connections of the cat's auditory cortex. Brain Res 212:1-15.

Kenet T, Froemke RC, Schreiner CE, Pessah IN, Merzenich MM (2007) Perinatal exposure to a noncoplanar polychlorinated biphenyl alters tonotopy, receptive fields, and plasticity in rat primary auditory cortex. Proc Natl Acad Sci U S A 104:7646-7651.

Kilgard MP, Merzenich MM (1998) Cortical map reorganization enabled by nucleus basalis activity. Science 279:1714-1718.

Kitzes LM, Hollrigel GS (1996) Response properties of units in the posterior auditory field deprived of input from the ipsilateral primary auditory cortex. Hear Res 100:120-130.

Knight PL (1977) Representation of the cochlea within the anterior auditory field (AAF) of the cat. Brain Res 130:447-467.

Kosaki H, Hashikawa T, He J, Jones EG (1997) Tonotopic organization of auditory cortical fields delineated by parvalbumin immunoreactivity in macaque monkeys. J Comp Neurol 386:304-316.

Kusmierek P, Rauschecker JP (2009) Functional specialization of medial auditory belt cortex in the alert rhesus monkey. J Neurophysiol 102: $1606-1622$.

Lee CC, Winer JA (2008) Connections of cat auditory cortex: III. Corticocortical system. J Comp Neurol 507:1920-1943.

Lomber SG (1999) The advantages and limitations of permanent or reversible deactivation techniques in the assessment of neural function. J Neurosci Methods 86:109-117.

Lomber SG, Malhotra S (2008) Double dissociation of "what" and "where" processing in auditory cortex. Nat Neurosci 11:609-616.

Lomber SG, Cornwell P, Sun JS, MacNeil MA, Payne BR (1994) Reversible inactivation of visual processing operations in middle suprasylvian cortex of the behaving cat. Proc Natl Acad Sci U S A 91:2999-3003.

Lomber SG, Payne BR, Horel JA (1999) The cryoloop: an adaptable reversible cooling deactivation method for behavioral or electrophysiological assessment of neural function. J Neurosci Methods 86:179-194.

Merabet L, Desautels A, Minville K, Casanova C (1998) Motion integration in a thalamic visual nucleus. Nature 396:265-268.

Merzenich MM, Knight PL, Roth GL (1973) Cochleotopic organization of primary auditory cortex in the cat. Brain Res 63:343-346.
Merzenich MM, Knight PL, Roth GL (1975) Representation of cochlea within primary auditory cortex in the cat. J Neurophysiol 38:231-249.

Middlebrooks JC, Pettigrew JD (1981) Functional classes of neurons in primary auditory cortex of the cat distinguished by sensitivity to sound location. J Neurosci 1:107-120.

Nakamoto KT, Jones SJ, Palmer AR (2008) Descending projections from auditory cortex modulate sensitivity in the midbrain to cues for spatial position. J Neurophysiol 99:2347-2356.

Noreña AJ, Gourévitch B, Pienkowski M, Shaw G, Eggermont JJ (2008) Increasing spectrotemporal sound density reveals an octave-based organization in cat primary auditory cortex. J Neurosci 28:8885-8896.

Olfert ED, Cross BM, McWilliam AA (1993) Guide to the care and use of experimental animals. Ottawa: Canadian Council on Animal Care.

Palmer AR, Hall DA, Sumner C, Barrett DJ, Jones S, Nakamoto K, Moore DR (2007) Some investigations into non-passive listening. Hear Res 229: 148-157.

Payne BR, Lomber SG (1996) Age-dependent modification of cytochrome oxidase activity in the cat dorsal lateral geniculate nucleus following removal of primary visual cortex. Vis Neurosci 13:805-816.

Phillips DP, Orman SS (1984) Responses of single neurons in posterior field of cat auditory cortex to tonal stimulation. J Neurophysiol 51:147-163.

Pons TP, Garraghty PE, Mishkin M (1992) Serial and parallel processing of tactual information in somatosensory cortex of rhesus monkeys. J Neurophysiol 68:518-527.

Rauschecker JP (1998) Parallel processing in the auditory cortex of primates. Audiol Neurootol 3:86-103.

Rauschecker JP, Tian B, Pons T, Mishkin M (1997) Serial and parallel processing in rhesus monkey auditory cortex. J Comp Neurol 382:89-103.

Reale RA, Imig TJ (1980) Tonotopic organization in auditory cortex of the cat. J Comp Neurol 192:265-291.

Rockland KS, Pandya DN (1979) Laminar origins and terminations of cortical connections of the occipital lobe in the rhesus monkey. Brain Res 179:3-20.

Rodman HR, Albright TD (1989) Single-unit analysis of pattern-motion selective properties in the middle temporal visual area (MT). Exp Brain Res 75:53-64.

Romanski LM, Tian B, Fritz J, Mishkin M, Goldman-Rakic PS, Rauschecker JP (1999) Dual streams of auditory afferents target multiple domains in the primate prefrontal cortex. Nat Neurosci 2:1131-1136.

Rose JE (1949) The cellular structure of the auditory region of the cat. J Comp Neurol 91:409-439.

Rouiller EM, Simm GM, Villa AE, de Ribaupierre Y, de Ribaupierre F (1991) Auditory corticocortical interconnections in the cat: evidence for parallel and hierarchical arrangement of the auditory cortical areas. Exp Brain Res 86:483-505.

Schmolesky MT, Wang Y, Hanes DP, Thompson KG, Leutgeb S, Schall JD, Leventhal AG (1998) Signal timing across the macaque visual system. J Neurophysiol 79:3272-3278.

Schreiner CE, Cynader MS (1984) Basic functional organization of second auditory cortical field (AII) of the cat. J Neurophysiol 51:1284-1305.

Sillito AM, Cudeiro J, Jones HE (2006) Always returning: feedback and sensory processing in visual cortex and thalamus. Trends Neurosci 29:307-316.

Sousa-Pinto A (1973) The structure of the first auditory cortex (A I) in the cat. I.-Light microscopic observations on its organization. Arch Ital Biol 111:112-137.

Van Essen DC, Felleman DJ, DeYoe EA, Olavarria J, Knierim J (1990) Modular and hierarchical organization of extrastriate visual cortex in the macaque monkey. Cold Spring Harb Symp Quant Biol 55:679-696.

Weinberger NM (2007) Auditory associative memory and representational plasticity in the primary auditory cortex. Hear Res 229:54-68.

Wessinger CM, VanMeter J, Tian B, Van Lare J, Pekar J, Rauschecker JP (2001) Hierarchical organization of the human auditory cortex revealed by functional magnetic resonance imaging. J Cogn Neurosci 13:1-7.

Winguth SD, Winer JA (1986) Corticocortical connections of cat primary auditory cortex (AI): laminar organization and identification of supragranular neurons projecting to area AII. J Comp Neurol 248:36-56.

Yang XF, Kennedy BR, Lomber SG, Schmidt RE, Rothman SM (2006) Cooling produces minimal neuropathology in neocortex and hippocampus. Neurobiol Dis 23:637-643. 